\title{
Identification of Disjoint Bi-decompositions in Boolean Functions through Walsh Spectrum
}

\author{
BOGDAN J. FALKOWSKI ${ }^{\mathrm{a}, *}$ and SUDHA KANNURAO ${ }^{\mathrm{b}}$ \\ ${ }^{a}$ School of Electrical and Electronic Engineering, Nanyang Technological University, Nanyang Avenue, Block S1, Singapore, Singapore 639798; \\ ${ }^{\mathrm{b}}$ Temasek Polytechnic, School of Engineering/Electronics, 21 Tampines Avenue 1, Singapore, Singapore 529757
}

(Received 1 May 2000; Revised 10 August 2000)

\begin{abstract}
A new algorithm for the identification of disjoint bi-decomposition in Boolean functions from its Walsh spectrum is proposed. The type of bi-decomposition and its existence is derived from the knowledge of a subset of Walsh spectrum for a Boolean function. All three types of bi-decomposition are considered including OR, AND and EXOR type. A filtering procedure that uses just few Walsh spectral coefficients (SC) is applied to quickly eliminate the functions that are not bi-decomposable and hence the algorithm is very efficient. The type of bi-decomposition and affirmation/negation of variables in its logic subfunctions are directly identified by manipulation on the reduced cubical representation of Boolean functions and their corresponding Walsh spectra. The presented algorithm has been implemented in $\mathrm{C}$ and tested on the standard benchmark functions. The number of Boolean functions having various disjoint bi-decompositions has also been enumerated.
\end{abstract}

Keywords: Boolean functions; Functional decomposition; Disjoint bi-decomposition; Walsh spectrum; Walsh transform

\section{INTRODUCTION}

Functional decomposition of Boolean functions allows decomposition of original logical system into sub-circuits that can be realized by some basic blocks from the library of various programmable logic devices. There are different types of functional decomposition and various methods for their identification [1-4,9-15]. The earlier classical methods used decomposition charts and the algebra of Boolean derivatives [1-4], while the recent methods are more powerful techniques that manipulate on reduced representations of Boolean functions such as decision diagrams or cubes [10-13]. Spectral methods based on Walsh transform of Boolean functions have also been used for identification of disjoint decomposition $[9,15]$ as well as to identify parallel and serial linear decomposition (extraction of linear pre- and post-filters) $[8,9]$. It should be noticed that the identification of a linear decomposition is only possible through the Walsh spectral domain.

Due to recent advances in programmable logic devices that have two-input logic elements in the outputs, an interest in bi-decomposition has developed. The methods that are used for this type of decomposition apply either decision diagrams or spectral representation of Boolean functions using the Reed-Muller transform [10,14]. For the first time, spectral conditions in the Walsh domain of the existence for bi-decomposition of Boolean functions were reported in Ref. [7]. In this paper, all types of bidecomposition are identified with exact determination of logic sub-functions with examples for each case. In majority of cases only a subset of the whole Walsh spectrum is necessary to either reject or confirm the existence of various types of bi-decomposition. The new algorithm has been implemented in $\mathrm{C}$ and tested on the standard benchmark functions. Finally, the number of Boolean functions having various types of considered bidecompositions have been given.

\section{PRELIMINARIES}

\section{Cubical Representation of a function}

An $n$-bit string is a vertex of an object called a 0 -cube. An $n$-variable Boolean function is represented as an

*Corresponding author. E-mail: efalkowski@ntu.edu.sg 


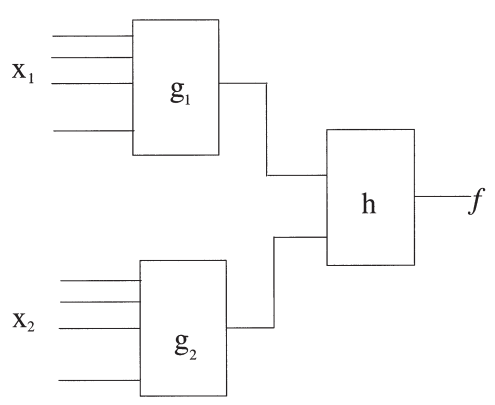

FIGURE 1 Disjoint bi-decomposition.

$n$-dimensional space ( $n$-hypercube) in which each vertex represents a minterm. A collection of $2^{i}, i \in\{0,1, \ldots, n\}$ adjacent minterms is called an $i$-cube [5]. A cube can be represented by an $n$-string of symbols 0,1 and -, where 0 corresponds to the negation of the variable, 1 to its affirmation value and - to the variable that is missing in the cube.

\section{Hadamard-Walsh Transform}

Let $f(x)$ denote a completely specified Boolean function over a set of $n$ input variables $X=\left(x_{1}, x_{2}, x_{3}, \ldots x_{n}\right), x_{u} \in$ $\{0,1\}, 1 \leq u \leq n$. The truth vector and the encoded $2^{n}$ output values of $f$ may be treated as column vectors $f \rightarrow$ and $F \rightarrow$ where the original elements of $f \rightarrow$ confined to $\{0,1\}$ are mapped to an $S$-coding of $F \rightarrow$ which is defined as [logic " $0 " \rightarrow 1]$ and [logic "1" $\rightarrow-1$ ], respectively. Then the Walsh-Hadamard spectrum $S \rightarrow$ of $f(x)$ is a column vector given by, $S \rightarrow=H^{n} F \rightarrow$ and $F \rightarrow=$ $\left(1 / 2^{n}\right) H^{n} S \rightarrow$ where $F \rightarrow=\left[f_{0}, f_{1}, f_{2}, \ldots, f_{2^{n}-1}\right]^{\mathrm{T}}$ and $S \rightarrow=\left[s_{0}, s_{1}, s_{2}, s_{12}, \ldots, s_{123 \ldots n}\right]^{\mathrm{T}}, \quad s_{1}$ are the spectral coefficients (SC) in Hadamard ordering and $I$ is the index of the coefficient of any order $o$, ranging from one to $n$. The DC coefficient or $s_{0}$ is related to the number of minterms for the function that have value 1 denoted by $a$ and its value is $s_{0}=2^{n}-2 a$. $H^{n}$ is the Hadamard-Walsh matrix or order $n[8]$.

\section{Disjoint Bi-decomposition}

Let $X=\left(X_{1}, X_{2}\right)$ be a partition of the variables representing a function $f(X)$ such that $X_{1} \cap X_{2}=0$ and $X_{1}$ is a group of $m$ variables and $X_{2}$ is a group of $n-m$ variables. $f\left(X_{1}, X_{2}\right)=h\left(g_{1}\left(X_{1}\right), g_{2}\left(X_{2}\right)\right)$ is said to be disjoint EXOR bi-decomposable if $f(X)$ can be realized as shown in Fig. 1 where $h$ is a two input EXOR gate and $g_{1}$ and $g_{2}$ are either AND, OR or EXOR gate. If $h$ is either AND or OR gate with $g_{1}$ and $g_{2}$ defined as above, then the

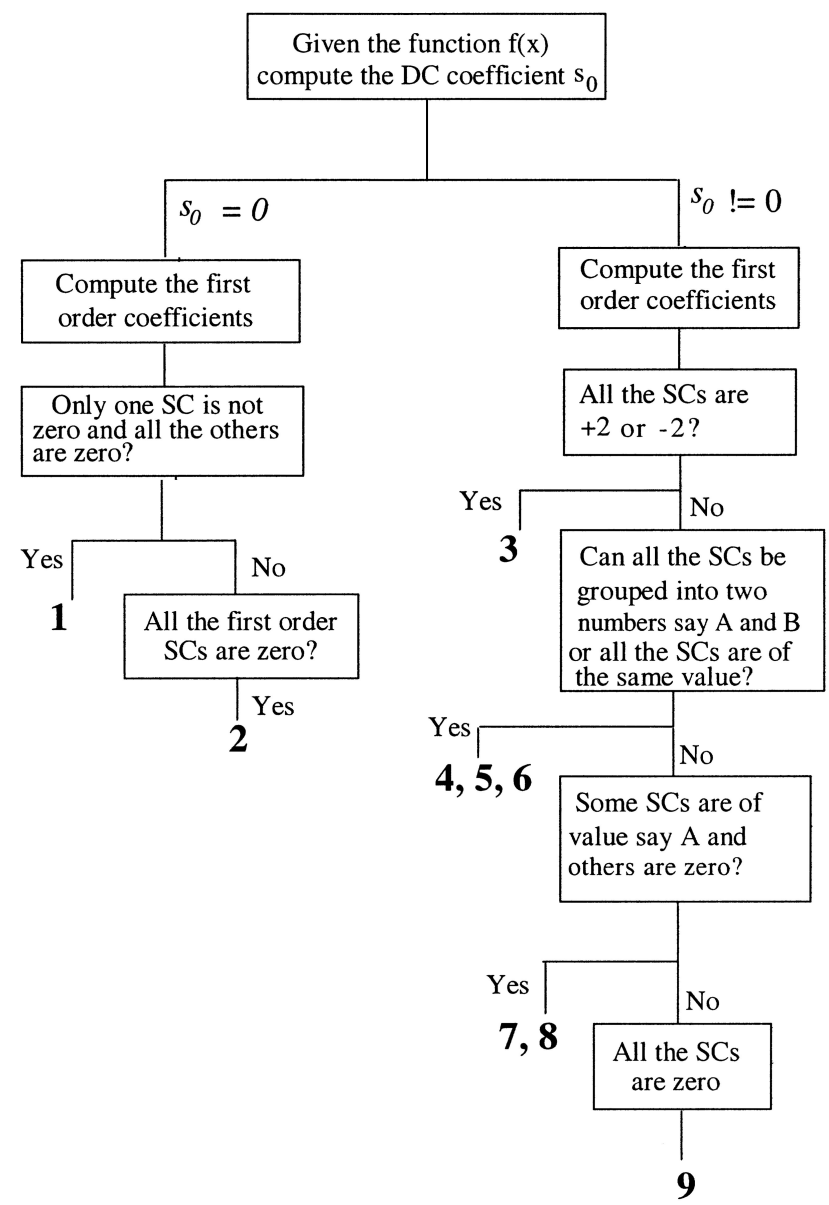

FIGURE 2 Flow chart to identify functions with bi-decomposition. 
decomposition is represented as disjoint AND or disjoint OR bi-decomposition, respectively [10-13].

\section{DISJOINT BI-DECOMPOSITION}

The flow chart in Fig. 2 shows the procedure to select a given function to test for the decomposition based on the DC coefficient and the first order Walsh SCs. In certain cases, where the first order SCs are all zero, the higher order coefficients need to be considered. If the preliminary conditions are satisfied, the algorithm discussed in the next section is applied on the selected function to identify the type of the decomposition. By this filtering procedure, the algorithm is very efficient and is effective in identifying the type and also the exact function from its cubical representation.

\section{ALGORITHM TO IDENTIFY TYPE OF DECOMPOSITION}

In the algorithm that is given below all the possible cases as outlined in the flow chart in Fig. 2 are discussed. The following three letters abbreviation scheme illustrated by an example is used throughout the algorithm for the type of the decomposition. The symbol AAE means that $g_{1}$ and $g_{2}$ are AND gates and $h$ the EXOR gate so that the output $f=\mathrm{a} \oplus \mathrm{b}$ (Fig. 1). Here A, E, O represent the AND, EXOR and OR gates, respectively, while symbols $\wedge, \oplus$ and $\vee$ represent the logical product, EXOR operation and logical sum, respectively.

\section{Case 1: (AAE)}

if $\left(\left(s_{0}=0\right)\right.$ and (one $\left.s_{i} \neq 0\right)$ and (all the other $\left.s_{i}=0\right)$ ) $\{j=2$; while $(j \leq n)\{$ Calculate all SCs for the order $j$; if $\left((i \in I)\right.$ and $\left(s_{1} \neq 0\right)$ and $\left.\left(\left|s_{1}\right|+\left|s_{i}\right|=2^{n}\right)\right)$

$j++$; else break; $\}$

if $(j=n+1)$

$\left\{\right.$ if $\left(s_{i}<0\right)\left\{f=\bar{x}_{i} \oplus\right.$ product of the remaining variables else if $\left(s_{i}>0\right)$

$\left\{f=x_{i} \oplus\right.$ product of the remaining variables $\left.\}\right\}$

Note: The second order coefficients identify if any variables in the product term are in negation.

Example 1 Consider the function $f\left(x_{4}, x_{3}, x_{2}, x_{1}\right)=$ $\Sigma(2,3,6,7,10,11,13,14) . s_{0}=0$; Calculating the first order coefficients, $s_{1}=s_{3}=s_{4}=0 ; s_{2}=12$. The values of the first order coefficients indicate that there is only one $\mathrm{SC}$ of first order that is not zero and all the other first order SCs are zero. As the values of the first order SCs satisfy the condition, further calculating the other order SCs, $s_{12}=$ $s_{23}=s_{24}=s_{1234}=4 ; \quad s_{123}=s_{124}=s_{234}=-4$. Hence any $\left|s_{1}\right|+\left|s_{i}\right|=2^{n}=16$. As the values have satisfied the conditions and as $s_{2}=12$, the function can be represented as: $x_{2} \oplus x_{1} x_{3} x_{4}$.

\section{Case 2: (EEE) \& (AEE)}

Case 2a: (EEE)

if $\left(\left(s_{0}=0\right)\right.$ and $\left.\left(s_{i}=0\right)\right)$

\{if $\left(n\right.$-th order $\left.\mathrm{SC}=2^{n}\right)\{f=\oplus$ of all the variables $\}$

else if $\left(n\right.$-th order $\left.\mathrm{SC}=-2^{n}\right)$

$\{f=\oplus$ of all the variables out of which odd number are in negation $\}$

Example 2 Consider the function $f\left(x_{4}, x_{3}, x_{2}, x_{1}\right)=$ $\Sigma(1,2,4,7,8,11,13,14)$. As $s_{0}=0$ and all the first order SCs are zero, and $s_{1234}=16$, the function is represented as: $x_{1} \oplus x_{2} \oplus x_{3} \oplus x_{4}$. As $s_{1234}$ is positive, there are no variables in negation in the representation of the function. Also this function can be represented as the EXOR sum of even number of negated variables.

Case 2b: (AEE)

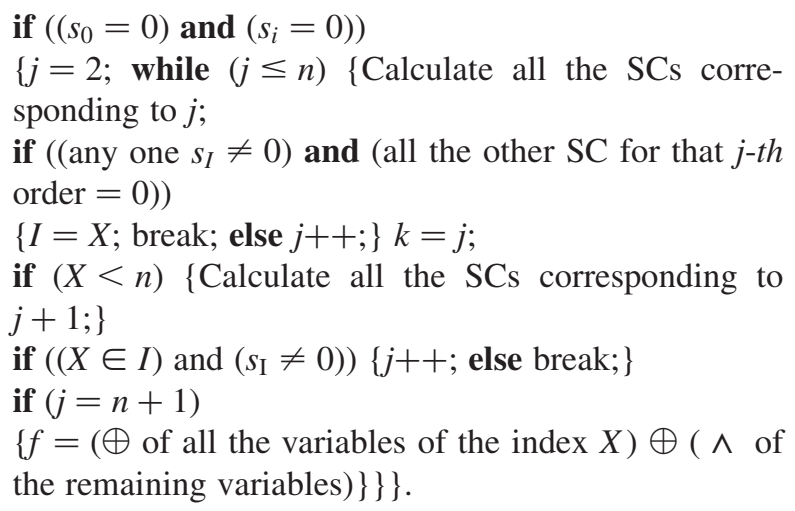

Example 3 A four variable function represented as, $f\left(x_{4}, x_{3}, x_{2}, x_{1}\right)=\Sigma(3,4,5,6,8,9,10,15)$ is considered. The DC coefficient and all the first order SCs are zero. Hence proceeding further to calculate the second order coefficients, $s_{34}=8$ and all the other SCs of second order are zero. Similarly, $s_{134}=s_{234}=8$ and $s_{1234}=-8$. All the other SCs are zero. As this function satisfies the condition for Case $2 b$, the function is represented as $x_{1} x_{2} \oplus\left(x_{3} \oplus x_{4}\right)$. Also as 34 is the index of the second order $\mathrm{SC}$ that is not zero, the variables $x_{3}$ and $x_{4}$ are represented as the EXOR function.

\section{Case 3: (AAA) \& (OOO)}

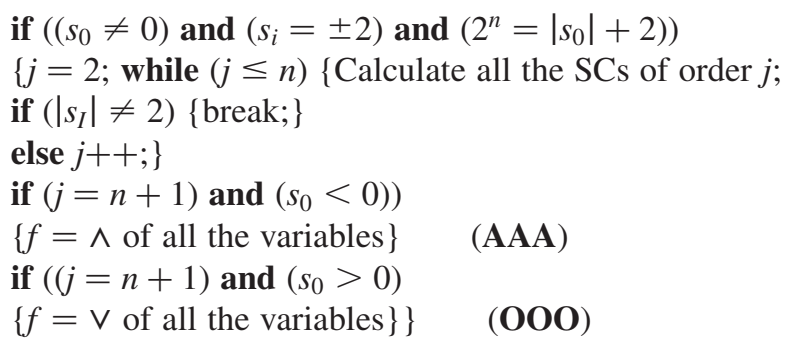

Note: $x_{i}$ is in negation for all $s_{i}=-2$. 
Example 4 Considering a four variable function, $f\left(x_{4}, x_{3}, x_{2}, x_{1}\right)=$

$\Sigma(1,2,3,4,5,6,7,8,9,10,11,12,13,14,15)$, the DC coefficient is -14 and all the other SC are 2. Neglecting the sign of the DC coefficient, $\left|s_{0}\right|+\mid$ any $s_{I} \mid=16$. Also as the DC coefficient is negative, the function is represented as $x_{1} \vee x_{2} \vee x_{3} \vee x_{4}$.

\section{Case 4: (AAO) \& (OOA)}

Case 4a:

if $\left(\left(s_{0} \neq 0\right)\right.$ and $\left(s_{i}= \pm \mathrm{X}\right)$ and $\left(2^{n}-\left|s_{0}\right|=(|\mathrm{X}|+\right.$ 1) $\left.{ }^{*} 2\right)$ and ( $n$ is even))

$\{j=2$; while $(j \leq n)$ \{Calculate all the SCs;

if $\left(\left(s_{I} \neq|\mathrm{X}|\right)\right.$ and $\left.\left(s_{I} \neq \pm 2\right)\right)$ \{break; $\}$

if $\left((j=n / 2)\right.$ and $\left.\left.\left(s_{I}=|\mathrm{X}|\right)\right)\{I=Y ; j++;\}\right\}$

if $(j=n+1)$ and $\left(s_{0}>0\right)$

$\{f=(\wedge$ all the variables of $Y) \vee(\wedge$ the remaining variables $) \quad$ (AAO)

if $\left((j=n+1)\right.$ and $\left.\left(s_{0}<0\right)\right)$

$\{f=(\vee$ all the variables of $Y) \wedge(\vee$ the remaining variables $)\}$ (OOA)

Note: $x_{i}$ is in negation for all $i$ where $s_{i}<0$.

Case 4b:

if $\quad\left(\left(s_{0} \neq 0\right) \quad\right.$ and $\quad\left(s_{i}= \pm \mathrm{X}\right.$ or $\left.\pm \mathrm{Y}\right) \quad$ and $\left.\left(2^{n}-\left|s_{0}\right|=|\mathrm{X}|+|\mathrm{Y}|+2\right)\right)$

$\{j=2$; while $(j \leq n)$ \{Calculate all the SCs;

if $\left(\left(s_{I} \neq|\mathrm{X}|\right)\right.$ and $\left(s_{I} \neq|\mathrm{Y}|\right)$ and $\left(s_{I} \neq \pm 2\right)$ \{break; else $j++;\}\}$

if $\left((j=n+1)\right.$ and $\left.\left(s_{0}>0\right)\right)$

$\left\{f=\left(\wedge\right.\right.$ all the variables whose $\left.s_{i}= \pm \mathrm{X}\right) \vee(\wedge$ all the variables whose $\left.s_{i}= \pm \mathrm{Y}\right)$

(AAO)

if $\left((j=n+1)\right.$ and $\left.\left(s_{0}<0\right)\right)$

$\left\{f=\left(\vee\right.\right.$ of all the variables whose $\left.s_{i}= \pm \mathrm{X}\right) \wedge(\vee$ of all the variables whose $\left.\left.\left.s_{i}= \pm \mathrm{Y}\right)\right\}\right\} \quad$ (OOA)

Note: Any $x_{i}$ is in negation if $s_{i}<0$.

Example 5 A four variable function $f\left(x_{4}, x_{3}, x_{2}, x_{1}\right)=$ $\Sigma(3,7,12,13,14,15)$ is considered. The DC coefficient is 2 and all the first order coefficients have the value 6. Further calculating the second order coefficients, $s_{12}=$ $s_{34}=-6$ and all the other second order SCs are 2. All the third and fourth order SC are -2 and 2 , respectively. Hence the function is represented as: $x_{1} x_{2} \vee x_{3} x_{4}$. This is an AAO decomposition from Case $4 \mathrm{a}$ since the DC spectral coefficient is greater than zero.

\section{Case 5: (AOA) \& (OAO)}

if $\left(\left(s_{0} \neq 0\right)\right.$ and $\left(s_{i}= \pm \mathrm{X} \quad\right.$ or $\left.\quad \pm 2\right)$ and $\left.\left(2^{n}=\left|s_{0}\right|+|\mathrm{X}|\right)\right)$ $\{j=2$; while $(j \leq n)$ \{Calculate all the SCs;

if $\left(\left(s_{I} \neq|\mathrm{X}|\right)\right.$ and $\left.\left(s_{I} \neq \pm 2\right)\right)$ \{break; else $\left.\left.j++;\right\}\right\}$

if $(j=n+1)$ and $\left(s_{0}>0\right)$

$\left\{f=\left(\wedge\right.\right.$ of all the variables whose $\left.s_{i}= \pm \mathrm{X}\right) \wedge(\vee$ of all the remaining variables)

(AOA)

if $\left((j=n+1)\right.$ and $\left.\left(s_{0}<0\right)\right)$

$\left\{f=\left(\vee\right.\right.$ of all the variables whose $\left.s_{i}= \pm \mathrm{X}\right) \vee(\wedge$ of all the remaining variables) $\}$ \}

(OAO)

Note: Any $x_{i}$ is in negation if $s_{i}<0$.

Example 6 Consider a four variable function given by: $f\left(x_{4}, x_{3}, x_{2}, x_{1}\right)=$

$\Sigma(1,2,3,5,6,7,9,10,11,12,13,14,15)$. The DC coefficient is -10 and the first order SCs with the index 1 and 2 are 6 and those with the index 3 and 4 are 2. Hence calculating the second order SCs $s_{12}=6$ and $s_{34}=-2$ and all the other SCs are 2. $s_{123}=s_{124}=2, s_{134}=s_{234}=$ $s_{1234}=-2$. Hence the function can be represented as $x_{1} \mathrm{~V}$ $x_{2} \vee x_{3} x_{4}$.

\section{Case 6: (AAE) \& (OAE)}

\section{Case 6a:}

if $\left(\left(s_{0} \neq 0\right)\right.$ and $\left(s_{i}= \pm \mathrm{X}\right)$ and $\left(2^{n}-\left|s_{0}\right|=(|\mathrm{X}|+\right.$ 2) $\left.{ }^{*} 2\right)$ and ( $n$ is even)

$\{j=2$; while $(j \leq n)$ \{Calculate all the SCs of order $j$;

if $\left(\left(s_{I} \neq|\mathrm{X}|\right)\right.$ and $\left.\left(s_{I} \neq 4\right)\right)$ \{break;

if $\left((j=n / 2)\right.$ and $\left.\left.\left(s_{I}=|\mathrm{X}|\right)\right)\{I=Y ; j++;\}\right\}$

if $(j=n+1)$ and $\left(s_{0}>0\right)$

$\{f=(\wedge$ of all the variables of $Y) \oplus(\wedge$ of the remaining variables)

(AAE)

if $\left((j=n+1)\right.$ and $\left.\left(s_{0}<0\right)\right)$

$\{f=(\vee$ of all the variables of $Y) \oplus(\wedge$ of the remaining variables) $\}\} \quad$ (OAE)

Note: (i) $x_{i}$ is in negation for all $s_{i}<0$. (ii) The AAE can also be represented as an OOE decomposition but the only change is that, that all the variables are complemented.

Case 6b:

if $\quad\left(\left(s_{0} \neq 0\right)\right.$ and $\quad\left(s_{i}= \pm \mathrm{X} \quad\right.$ or $\left.\quad \pm \mathrm{Y}\right) \quad$ and $\left.\left(2^{n}-\left|s_{0}\right|=|\mathrm{X}|+|\mathrm{Y}|+4\right)\right)$

$\{j=2$; while $(j \leq n)$ \{Calculate all the SCs of order $j$; if $\left(\left(s_{I} \neq|\mathrm{X}|\right)\right.$ and $\left(s_{I} \neq|\mathrm{Y}|\right)$ and $\left(s_{I} \neq \pm 4\right)$ and $(j \leq$ $n / 2+1)$ and ( $n$ is even $))$ \{break;

if $\left(\left(s_{I} \neq \pm 4\right)\right.$ and $(j>n / 2+1)$ and $(n$ is even $\left.)\right)$ $\{$ break; $\}$

if $\left(\left(s_{I} \neq|\mathrm{X}|\right)\right.$ and $\left(s_{I} \neq|\mathrm{Y}|\right)$ and $\left(s_{I} \neq \pm 4\right)$ and $(j \leq$ $n+1 / 2)$ and $(n$ is odd $))\{$ break; $\}$

if $\left(\left(s_{I} \neq \pm 4\right)\right.$ and $(j>(n+1) / 2)$ and $(n$ is odd $\left.)\right)$ $\{$ break; $\} j++;\}$

if $(j=n+1)$ and $\left(s_{0}>0\right)$

$\left\{f=\left(\wedge\right.\right.$ of all the variables whose $\left.s_{i}= \pm \mathrm{X}\right) \oplus(\wedge$ of all the variables whose $\left.s_{i}= \pm \mathrm{Y}\right)$

(AAE)

if $(j=n+1)$ and $\left(s_{0}<0\right)$ 
TABLE I The possible number of decompositions of a specific type for Boolean functions

\begin{tabular}{llcc}
\hline Type of decomposition & \# of such decompositions possible & Type of decomposition & \# of such decompositions possible \\
\hline OOA, AAO & $\sum_{i=1}^{m} C_{i}^{n} 2^{n}$ & AOO & $\sum_{i=2}^{n-2} C_{i}^{n} 2^{n}$ \\
AEA, OEA, OEO, AEO & $\sum_{i=1}^{n-2} C_{i}^{n} 2^{i+1}$ & EEA, EEO & $4 \sum_{i=2}^{n} C_{i}^{n}$ \\
AOA & $\sum_{i=1}^{n=2} C_{i}^{n} 2^{n}$ & AEE & $\sum_{i=2}^{n} C_{i}^{n} 2^{i+1}$ \\
AAE & $n 2^{n}$ & AAA, OOO & $2^{n}$ \\
EEE & 2 & & \\
\hline
\end{tabular}

$\left\{f=\left(\mathrm{\vee}\right.\right.$ of all the variables whose $\left.s_{i}= \pm \mathrm{X}\right) \oplus(\wedge$ of all the variables whose $\left.\left.\left.s_{i}= \pm \mathrm{Y}\right)\right\}\right\} \quad$ (OAE)

Note: Any $x_{i}$ is in negation if $s_{i}<0$.

Example 7 Consider a four variable function given by: $f\left(x_{4}, x_{3}, x_{2}, x_{1}\right)=\Sigma(3,7,11,12,13,14)$. The DC coefficient is 4 and as all the other SCs are 4 or -4 , which follows all the conditions as outlined for Case $6 a$, the function can be represented as $x_{1} x_{2} \oplus x_{3} x_{4}$.

\section{Case 7: (AEA) \& (OEO)}

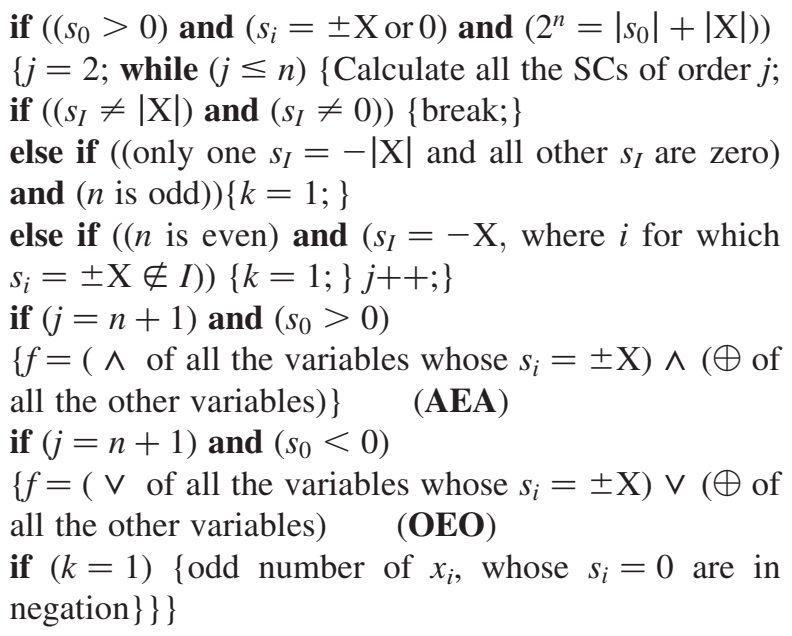

Note: Any $x_{i}$ is in negation if $s_{i}<0$.

Example 8 The four variable function is represented as: $f\left(x_{4}, x_{3}, x_{2}, x_{1}\right)=\Sigma(1,2,3,4,5,6,7,8,9,10,11,13,14,15)$. The DC coefficient is -12 and further calculating the first order coefficients, $s_{1}=s_{2}=4 ; s_{3}=s_{4}=0$; The second order coefficients are: $s_{12}=s_{34}=4$ and all the other SCs are zero. Similarly, the other coefficients that are non zero of third and fourth order are $s_{134}, s_{234}$ and $s_{1234}$. As the conditions outlined for Case 7 are satisfied, the function can be represented as $\left(x_{1} \vee x_{2}\right) \vee\left(x_{3} \oplus x_{4}\right)$.

\section{Case 8: (OEA) \& (AEO)}

$$
\begin{aligned}
& \text { if }\left(\left(s_{0} \neq 0\right) \text { and }\left(s_{i}= \pm \mathrm{X} \text { or } 0\right) \text { and }\left(s_{0}=|A|\right)\right) \\
& \left\{j=2 ; \quad \mathrm{Y}=2^{n}-|\mathrm{X}| \text {; while }(j \leq n)\{\text { Calculate all }\right. \\
& \text { the } \mathrm{SCs} ; \\
& \text { if } \left.\left(\left(s_{I} \neq|\mathrm{X}|\right) \text { and }\left(s_{I}=0\right) \text { and }\left(s_{I} \neq|\mathrm{Y}|\right)\right) \text { \{break; }\right\} \\
& \text { else if }\left(( \text { only one } s _ { I } = - | \mathrm { Y } | ) \text { and } \left(\text { all other } s_{I}\right.\right. \text { are zero or } \\
& \pm \mathrm{X})) \\
& k=1 ; j++;\} \\
& \text { if }\left((j=n) \text { and }\left(s_{0}+\mathrm{Y}=2^{n}\right) \text { and }\left(s_{0}>0\right)\right) \\
& \left\{f=\left(\vee \text { of all the variables whose } s_{i}= \pm \mathrm{X}\right) \wedge(\oplus \text { of }\right. \\
& \text { all the other variables) }(\text { OEA) } \\
& \text { if }\left((j=n) \text { and }\left(s_{0}+\mathrm{Y}=2^{n}\right) \text { and }\left(s_{0}<0\right)\right) \\
& \left\{f=\left(\wedge \text { of all the variables whose } s_{i}= \pm \mathrm{X}\right) \vee(\oplus \text { of all }\right. \\
& \text { the other variables })\} \quad(\text { AEO) } \\
& \text { if }(k=1)\left\{\text { odd number of } x_{i}, \text { whose } s_{i}=0\right. \text { are in } \\
& \text { negation }\}\}\}
\end{aligned}
$$

Note: Any $x_{i}$ is in negation if $s_{i}<0$.

Example 9 The four variable function is represented as: $f\left(x_{4}, x_{3}, x_{2}, x_{1}\right)=\Sigma(5,6,7,9,10,11)$. The DC coefficient is 4 and the first order coefficients, $s_{1}=s_{2}=4 ; s_{3}=$ $s_{4}=0$; The non zero second order coefficients are: $s_{12}=$ 4 and $s_{34}=12$ and all the other SC are zero. The third and fourth order non zero SCs are $s_{134}, s_{234}$, and $s_{1234}$ and their value are -4 . As the conditions outlined for Case 8 are

TABLE II Experimental results

\begin{tabular}{lccc}
\hline Standard benchmark function & No. of inputs & No. of outputs & Run time (s) \\
\hline Xor5 & 5 & 1 & 0.03 \\
Cm138a & 6 & 8 & 0.03 \\
Ex5 & 8 & 63 & 0.35 \\
Exps & 8 & 38 & 0.21 \\
Luc & 8 & 27 & 0.04 \\
Rd84 & 8 & 4 & 0.04 \\
Br1 & 12 & 8 & 0.21 \\
Br2 & 12 & 8 & 0.18 \\
\hline
\end{tabular}


satisfied, the function is represented as $\left(x_{1} \vee x_{2}\right) \wedge$ $\left(x_{3} \oplus x_{4}\right)$

\section{Case 9: (EEA) \& (EEO)}

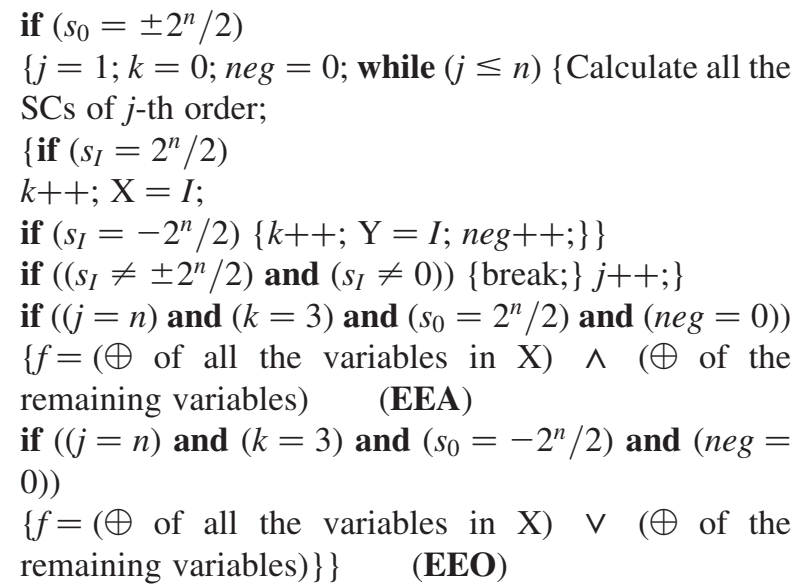

Note: Odd number of variables in $g_{2}$ are in negation if $n e g=1$, odd number of variables in both the groups $g_{1}$ and $g_{2}$ are in negation if $n e g=2$.

Example 10 The four variable function is represented as: $f\left(x_{4}, x_{3}, x_{2}, x_{1}\right)=\Sigma(5,6,9,10)$. The DC coefficient is 8 and all the first order coefficients are zero. The non zero second order coefficients are: $s_{12}=s_{34}=8$ and all the other $\mathrm{SC}$ are zero. The fourth order non zero $\mathrm{SC}$ is -8 . As the conditions outlined for Case 9 are satisfied, the function is represented as $\left(x_{1} \oplus x_{2}\right) \wedge\left(x_{3} \oplus x_{4}\right)$.

\section{ENUMERATION OF BI-DECOMPOSITIONS}

For any given $n$-variable Boolean function the number of decompositions of a particular type is listed in the Table I.

\section{EXPERIMENTAL RESULTS}

The algorithm described in the "Algorithm to identify type of decomposition" section was implemented in $\mathrm{C}$ and run on the HP 9000 workstation. The standard benchmark logic functions have been considered for testing. The results are shown in Table II. Columns in Table II from left to right represent the benchmark function, the number of input variables for that function, the run time in seconds, and the number of outputs of the same benchmark function that has any one of the bi-decompositions discussed here.

\section{CONCLUSION}

Spectral conditions in the Walsh domain for the identification of existence and type of the bi-decomposition for Boolean functions have been presented. The new algorithm is efficient in finding the type of bidecomposition and its exact logical sub-functions. By using a filtering procedure, the algorithm can eliminate the lack of existence of the bi-decomposition without the necessity of calculating and operating on the whole Walsh spectrum. This work extended the Walsh spectral theory of standard types of decomposition known earlier $[6,8,9,15]$ to the bi-decomposition. Consideration of various types of bi-decomposition is important not only from theoretical but also from practical point of view as there are many recent programmable logic devices that can be efficiently used for direct implementation of types of bi-decompositions discussed in this article.

\section{References}

[1] Ashenhurst, R. L. (1957). "The decomposition of switching functions", In Proc. Int. Symp. on the Theory of Switching Functions, April, pp. 74-116.

[2] Bliznyuk, V.D. and Kholodnyi, M.F. (1984) "Decomposition of Boolean functions applying the apparatus of Boolean derivatives", Automata and Remote Control, 636-643.

[3] Bochmann, D., Dresig, F. and Steinbach, B. (1991) "A new decomposition method for multilevel circuit design", Proc. EDAC February, 374-377.

[4] Curtis, H.A. (1962) A New Approach to the Design of Switching Circuits (Van Nostrand, New York).

[5] DeMicheli, G. (1994) Synthesis and Optimization of Digital Circuits (McGraw-Hill, New York).

[6] Diaz-Olavarrieta, L., Illanko, K. and Zaky, S.G. (1993) "Goaloriented decomposition of switching functions", IEEE Trans. Comput.-Aided Design Integrated Circuits Syst. 12(5), 655-665.

[7] Falkowski, B.J. and Kannurao, S. (2000). "Efficient spectral method for disjoint bi-decomposition of Boolean functions", Proc. 33rd IEEE Int. Symp. on Circuits and Systems, Geneva, Switzerland, Vol. 2, pp. 313-316.

[8] Karpovsky, M.G. (1976) Finite Orthogonal Series in Design of Digital Devices (Wiley, New York).

[9] Poswig, J. (1991) "Disjoint decomposition of Boolean functions", IEE Proc. $-E$ 138(1), 48-56.

[10] Sasao, T. and Butler, J.T. (1997). "On bi-decomposition of logic functions", Notes of Int. workshop on Logic Synthesis, May.

[11] Sawada, H., Yamashita, S. and Nagoya, A. (1997). "Restricted simple disjunctive decompositions based on grouping symmetric variables", 7th Great Lakes Symp. On VLSI, pp. 39-44.

[12] Sawada, H., Yamashita, S. and Nagoya, A. (1998). "Restructing logic representations with easily detectable simple disjunctive decompositions", Proc. Design, Automation and Test in Europe Conf., pp. 755-759, February.

[13] Yamashita, S., Sawada, H. and Nagoya, A. (1993). "New methods to find optimal non-disjoint bi-decompositions", Proc. Int. Conf. Computer-Aided Design, November, pp. 680-684.

[14] Tran, A. and Lin, T-C. (1994) "Simple disjoint decomposition of Reed-Muller polynomials", Elect. Lett. 30(6), 468-469.

[15] Tokmen, V.H. (1980). "Disjoint decomposability of multi-valued functions by spectral means", Proc. IEEE 10th Int. Symp. MultipleValued Logic, pp. 88-93.

\section{Authors' Biographies}

Bogdan J. Falkowski received the MSEE degree from Technical University of Warsaw, Poland and the PhD 
degree in Electrical and Computer Engineering from Portland State University, Oregon, USA. His industrial experience includes research and development positions at several companies. He then joined the Electrical and Computer Engineering Department at Portland State University. Since 1992 he has been with the School of Electrical and Electronic Engineering, Nanyang Technological University in Singapore where he is currently an Associate Professor. His research interests include VLSI systems and design, switching circuits, testing, spectral methods, and design of algorithms. He has published three book chapters and over 180 refereed journal and conference articles. He is a senior member of the IEEE and technical chair for International Conference on Information, Communication and Signal Processing hold in December 1999.

Sudha Kannurao received the M.Eng. (by research) degree in Electrical Engineering from Nanyang Technological University, Singapore in 2000. She had been working as a development Engineer and also as lecturer from 1989 to 1998 . Currently she is a faculty member in School of Engineering, Temasek Polytechnic, Singapore. Her research interests include logic synthesis, VLSI systems and design using various spectral techniques. 

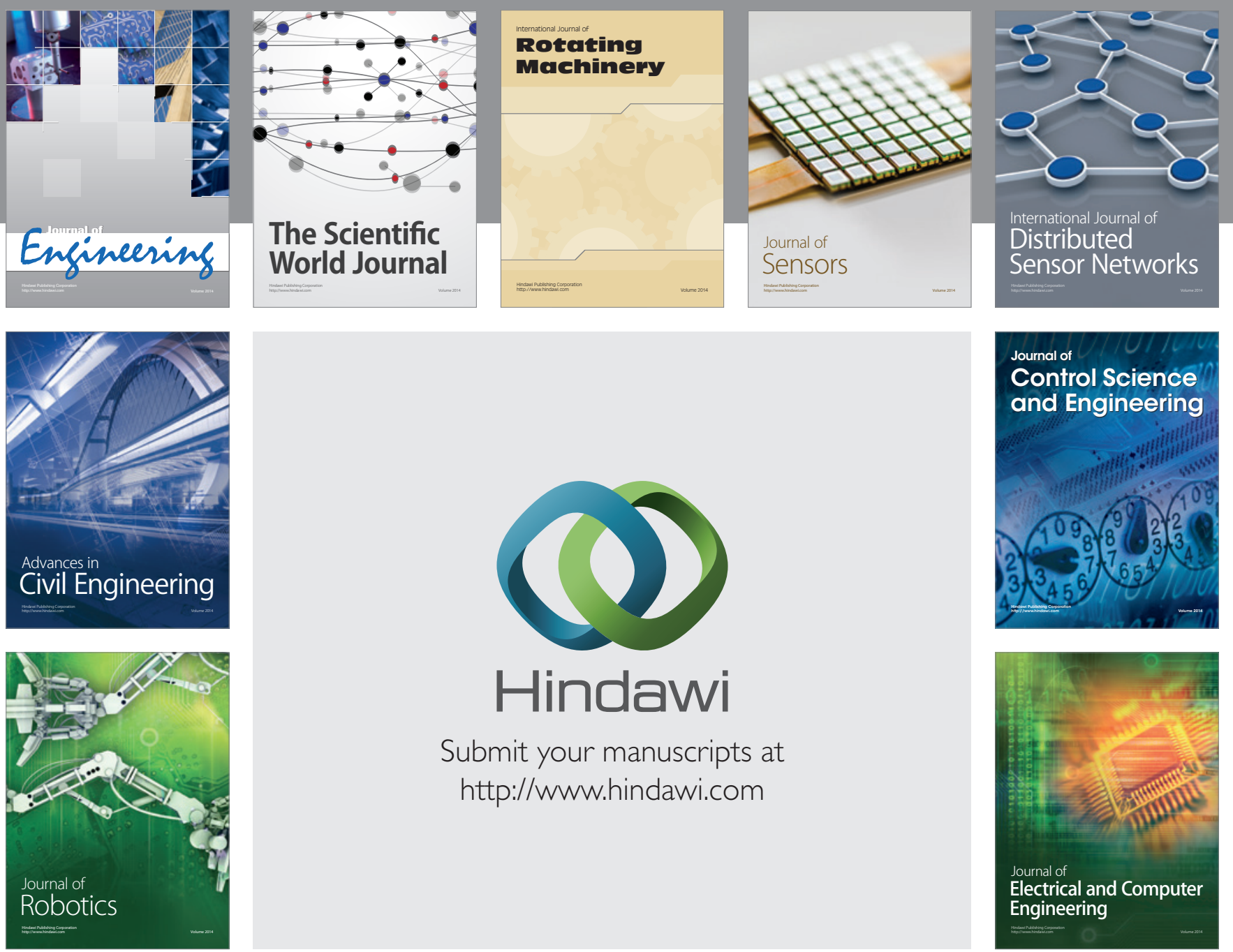

Submit your manuscripts at

http://www.hindawi.com
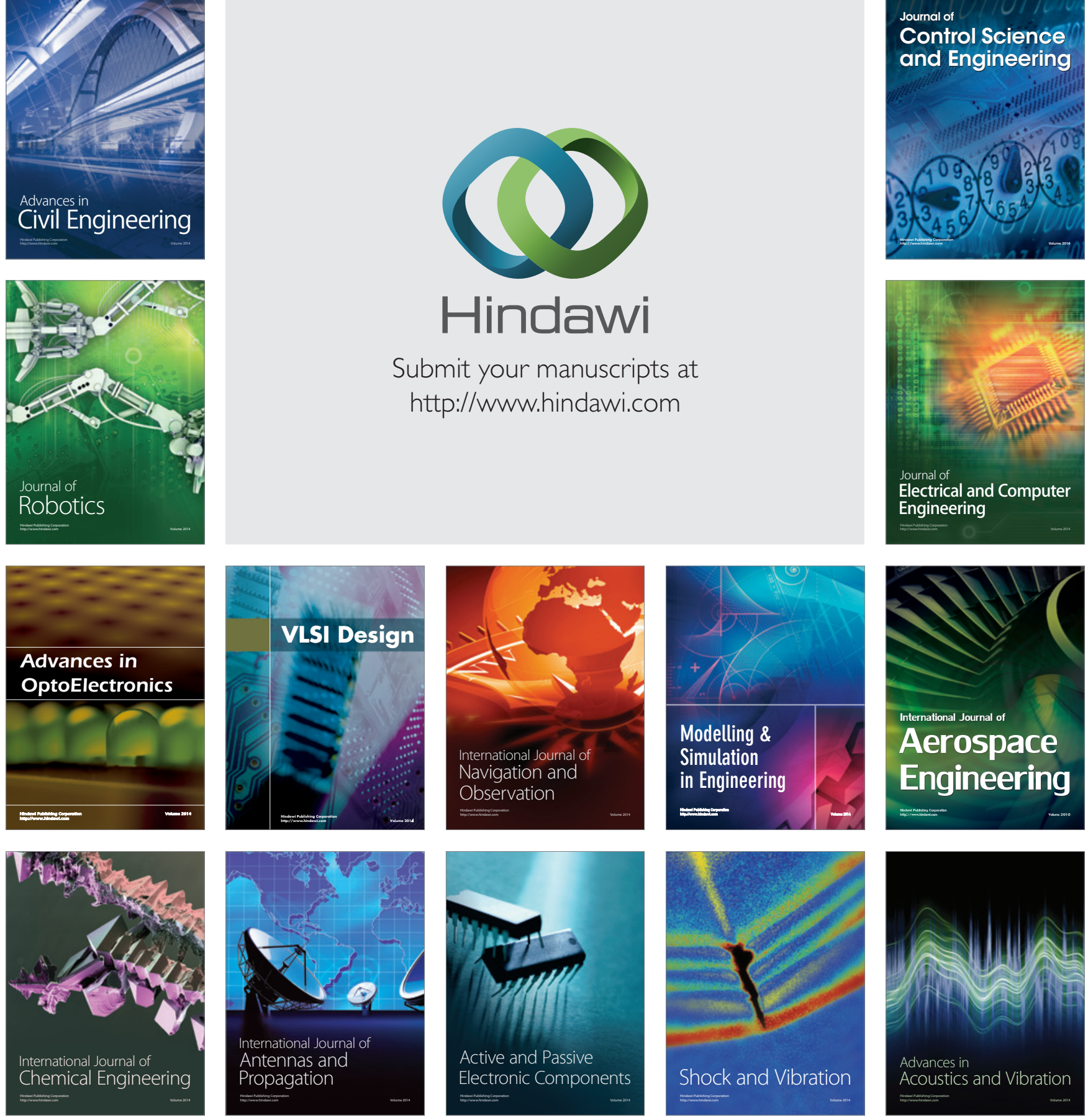